\title{
Comparison of gait analysis between a triaxial accelerometer- based device and an optical motion capture system
}

\author{
Taisuke Ito $^{1 \S}$, Yuichi Ota ${ }^{1}$ \\ ${ }^{1}$ Waseda Elderly Health Association Co., Ltd., 4-24-58 Takanawa, Minato-ku, Tokyo-to 108-0074, Japan \\ ${ }^{\S}$ Corresponding author: \\ Taisuke Ito \\ Tel.: +81-3-5447-5470 \\ E-mail address: t ito@waseda-e-life.co.jp \\ ORCID iD: https://orcid.org/0000-0003-2080-1000
}

\begin{abstract}
AYUMI EYE is an accelerometer-based gait analysis device that measures the $3 \mathrm{D}$ accelerations of the human trunk. This study investigated the measurement accuracy of the AYUMI EYE as hardware as well as the accuracy of the gait cycle extraction program via simultaneous measurements using AYUMI EYE, a ground reaction force (GRF), and an optical motion capture system called VICON. The study was conducted with four healthy individuals as participants. The gait data were obtained by simulating four different patterns for three trials each: normal walking, anterior-tilt walking, hemiplegic walking, and shuffling walking. The AYUMI EYE and VICON showed good agreement for both the acceleration and displacement data. The durations of subsequent stride cycles calculated using the AYUMI EYE and GRF were in good agreement based on the calculated cross-correlation coefficients (CCs) with an $r$ value of 0.896 and p-value less than 0.05 , and their accuracies for these results were sufficient.
\end{abstract}

Keywords - AYUMI EYE, acceleration sensor, gait analysis, optical sensor

\section{INTRODUCTION}

Over the past few years, various gait analysis devices have been developed and used in medical and nursing care, as well as in other monitoring situations. This development is important because maintaining the ability to walk and the independence in walking are related to the quality of life as well as the extension of healthy life expectancy and survival [1-3]. The gait analysis device AYUMI EYE (Waseda Elderly Health Association Co., Ltd.) [4] is a device that can score the gait function instantly, based on acceleration data during walking, using a triaxial accelerometer and a gait cycle extraction program embedded in an iOS application. This program predicts the durations of the subsequent stride cycles, utilizing foot contacts detected via a segmentation algorithm. In gait analysis using an accelerometer, the gait motion is considered as a periodic movement of the body's center of gravity (COG), and the rhythmic change of COG is measured using an accelerometer, which allows objective measurements of the gait abnormalities [5-6]. Gait analysis using a single point accelerometer has been studied in various scenarios, such as comparing young and 
elderly subjects, verifying the relevance of general clinical examinations, and application to different subjects such as healthy adults and stroke patients [5-13]. Moreover, good reproducibility and validity have been reported in analyses where the accelerometer was attached near the lumbar portion of the spine [5][1315]. Gait analysis using accelerometers is expected to be widely utilized in clinical settings because of the simplicity, low cost, and unconstrained measurement environment of the accelerometer compared to conventional non-accelerometer gait analysis equipment [16]. In the AYUMI EYE device, data processing can be performed automatically, thereby making it more useful in many situations.

In AYUMI EYE, the gait parameters are defined using triaxial acceleration data obtained from the areas around the lumbar region of the spine [8-13], and the reproducibility of the parameters is sufficient to use in clinical situations [17]. However, the accuracy of the AYUMI EYE data and the durations of the subsequent stride cycles obtained automatically from the data were not sufficiently clarified.

This study was conducted to investigate the measurement accuracy of AYUMI EYE as a device and the gait cycle extraction program by simultaneous measurement and comparison of the data from AYUMI EYE, the ground reaction force (GRF) data, and the marker displacements of an optical motion capture system (VICON).

\section{METHODS}

Four healthy participants ( 3 males and 1 female, age: 30-70), who had no diseases or conditions that were thought to affect their ability to walk, participated in this study (mean \pm standard deviation (SD), height: $162.5 \pm 6.5 \mathrm{~cm}$; body mass: $66.3 \pm 18.9 \mathrm{~kg}$ ). The experimental protocol was approved by the Institutional Review Board of GE Healthcare Japan Corporation (IRB-OT-2015-002). All the participants provided their written informed consent before participation.

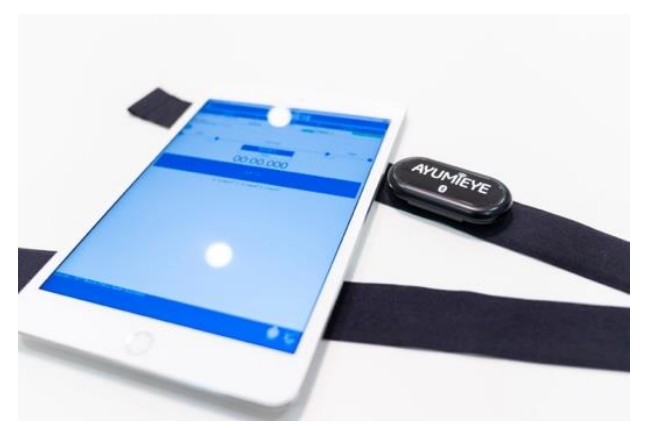

Fig 1. AYUMI EYE: A triaxial accelerometer with an iOS application

The AYUMI EYE (Fig. 1, sampling frequency: $31.25 \mathrm{~Hz}$ ), a force plate (AMTI, sampling frequency: $100 \mathrm{~Hz}$ ), and an optical motion capture system called VICON Nexus (VICON Motion Systems Ltd., 10 cameras, sampling frequency: $100 \mathrm{~Hz}$ ) were used as the gait analysis instruments. The participants using the AYUMI EYE module walked in a laboratory room outfitted with a force plate and the VICON 
system. A retro-reflective marker was placed in the same position as the AYUMI EYE module, which was attached to a rubber belt and adjusted to align with the third lumbar vertebra that approximates the COG of the human body (Fig. 2). The weight of the AYUMI EYE module is $18.5 \mathrm{~g}$ (including the battery) and its dimensions are $62.4 \times 30.9 \times 11.8 \mathrm{~mm}$. The built-in triaxial accelerometer in the AYUMI EYE module detects acceleration along the $\mathrm{x}$ (longitudinal), $\mathrm{y}$ (lateral), and $\mathrm{z}$ (vertical) axes of the device's coordinate system. These acceleration signals were acquired, digitized, and transmitted to a tablet (iPad, Apple Inc.) using Bluetooth communication. The iOS application designed for use with the module receives these data and computes the parameters related to the gait. In particular, the duration of the subsequent stride cycles is estimated using a program that extracts time information using the periodicity of the acceleration waveforms. As the AYUMI EYE is an accelerometer, the researchers did not have to perform any preexperimental calibration. Zero-point correction based on the gravitational acceleration was performed every time the system was turned on. Acceleration data obtained with the AYUMI EYE were low-pass filtered before further analysis.

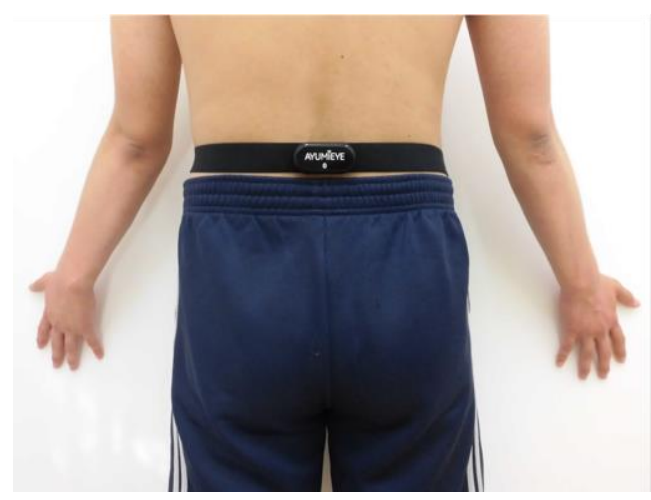

Fig. 2. AYUMI EYE placement location on the research participant

To evaluate whether the AYUMI EYE can be used for measurements under all gait conditions, gait data were obtained for four different pattern simulations with three experimental trials per pattern: normal walking, anterior-tilt walking (posture with approximately $25^{\circ}$ of trunk flexion from the vertical) [18], hemiplegic walking (mimicking the characteristics of a hemiplegic patient who can walk) [19], and shuffling walking (decreased height of toes off the floor during the swing phase) [20]. The order of the experimental trials in the four conditions was randomized, and all trials were performed after sufficient warm-up.

The AYUMI EYE data were collected approximately 15 steps from the beginning to the end of measurements. The GRF data were analyzed according to the gait cycle of steady-state walking. The first contact with the force plate was performed at the fifth step from the start of walking, and this instant was defined as time 0 . The VICON data were synchronized with those of the force plate using an analog-todigital board, and information was collected for approximately 10 additional steps. The AYUMI EYE was 
synchronized with the other instruments after measurements using the acquired data. The GRF and AYUMI EYE data were first synchronized by matching the timing of the waveform peaks of the vertical component of the GRF with the vertical acceleration during the stance phase. Displacement values were estimated by time integration from the acceleration data acquired by the AYUMI EYE, and the trend was obtained by a moving average processing step in the time domain with the VICON displacement data; further, the lowfrequency fluctuations were removed from the original signal, and the accuracy of agreement between the acceleration and displacement waveforms of the AYUMI EYE and VICON was improved by preprocessing with a Fourier-domain high-pass filter. The time information in the waveforms of the AYUMI EYE and VICON displacement data was confirmed to be in general agreement and was indirectly synchronized. The VICON camera and force plate data were low-pass filtered at $6 \mathrm{~Hz}$ (camera) and $18 \mathrm{~Hz}$ (force plate) before further analysis. The data were then compared in the following three manners.

\subsection{Comparison of the acceleration data of AYUMI EYE and VICON}

The cross-correlation coefficients (CCs) [21] were calculated for all trials as indicators of the degree of agreement between the 3D acceleration data of the AYUMI EYE and the 3D acceleration obtained from the second-order differentiation of the 3D marker displacement coordinates of the VICON. The significance level was set at $\mathrm{p}<0.05$. The mean and standard deviations (SD) of the CCs of all gait data were calculated for each axis.

\subsection{Comparison of displacement data of AYUMI EYE and VICON}

The CCs were calculated for all trials using the 3D displacement data obtained from the second-order integration of the 3D acceleration data from the AYUMI EYE and the 3D marker displacement coordinates of VICON. The significance level was set at $\mathrm{p}<0.05$, and the mean and SD of the CCs of all gait data were calculated for each axis.

\subsection{Comparison of durations of subsequent stride cycles of AYUMI EYE and force plate}

The CCs were calculated for all trials using the durations of the subsequent stride cycles obtained from the AYUMI EYE gait cycle extraction program and the force plate. The significance level was set at $p<0.05$, and all gait cycle information obtained from the force plate was included in the analysis. For the calculation of the durations of subsequent stride cycles of the force plate, the instant at which the vertical GRF increased from zero to a positive value was defined as the foot ground contact, and the instant at which the vertical GRF changed from a positive value to zero was defined as the foot off.

\section{RESULTS}




\subsection{Acceleration data of AYUMI EYE and VICON}

Figure 3 shows a typical example of the comparison between the acceleration data of the AYUMI EYE and those calculated from marker displacements. For each peak of the acceleration waveform, the AYUMI EYE showed a tendency for larger values (sharper peaks) than the VICON, while other parts of the waveform were in good agreement. The CCs were significant for each axis for all gait data. The mean and SD of the $\mathrm{CC}$ of the $3 \mathrm{D}$ acceleration were high [vertical axis, a_z $=0.926 \pm 0.055$; lateral axis, a $\_\mathrm{y}=0.834 \pm 0.259$; longitudinal axis, a_x $=0.689 \pm 0.219$ (Fig. 5)].

\subsection{Displacement data of AYUMI EYE and VICON}

Figure 4 shows a typical example of the comparison between the displacement data calculated from AYUMI EYE and those of the marker. The two waveforms are observed to be in good agreement. The CCs were significant along each axis for all gait data. The mean and SD of the CC of the 3D displacements were high [vertical axis, $\mathrm{z}=0.977 \pm 0.021$; lateral axis, $\mathrm{y}=0.898 \pm 0.245$; longitudinal axis, $\mathrm{x}=0.754 \pm 0.272$ (Fig. 5)].

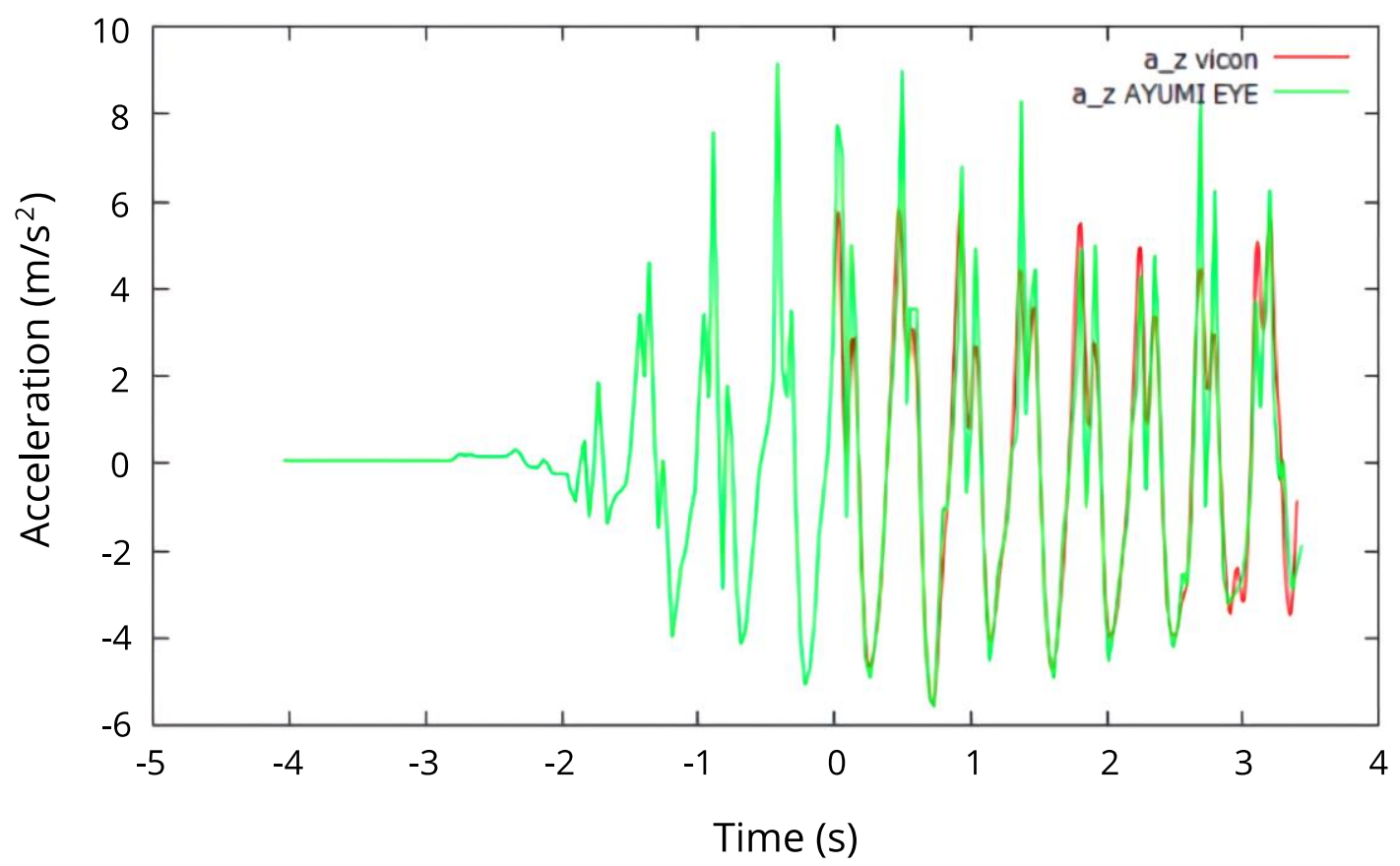

Fig. 3. Typical examples of vertical acceleration waveforms from AYUMI EYE and VICON 


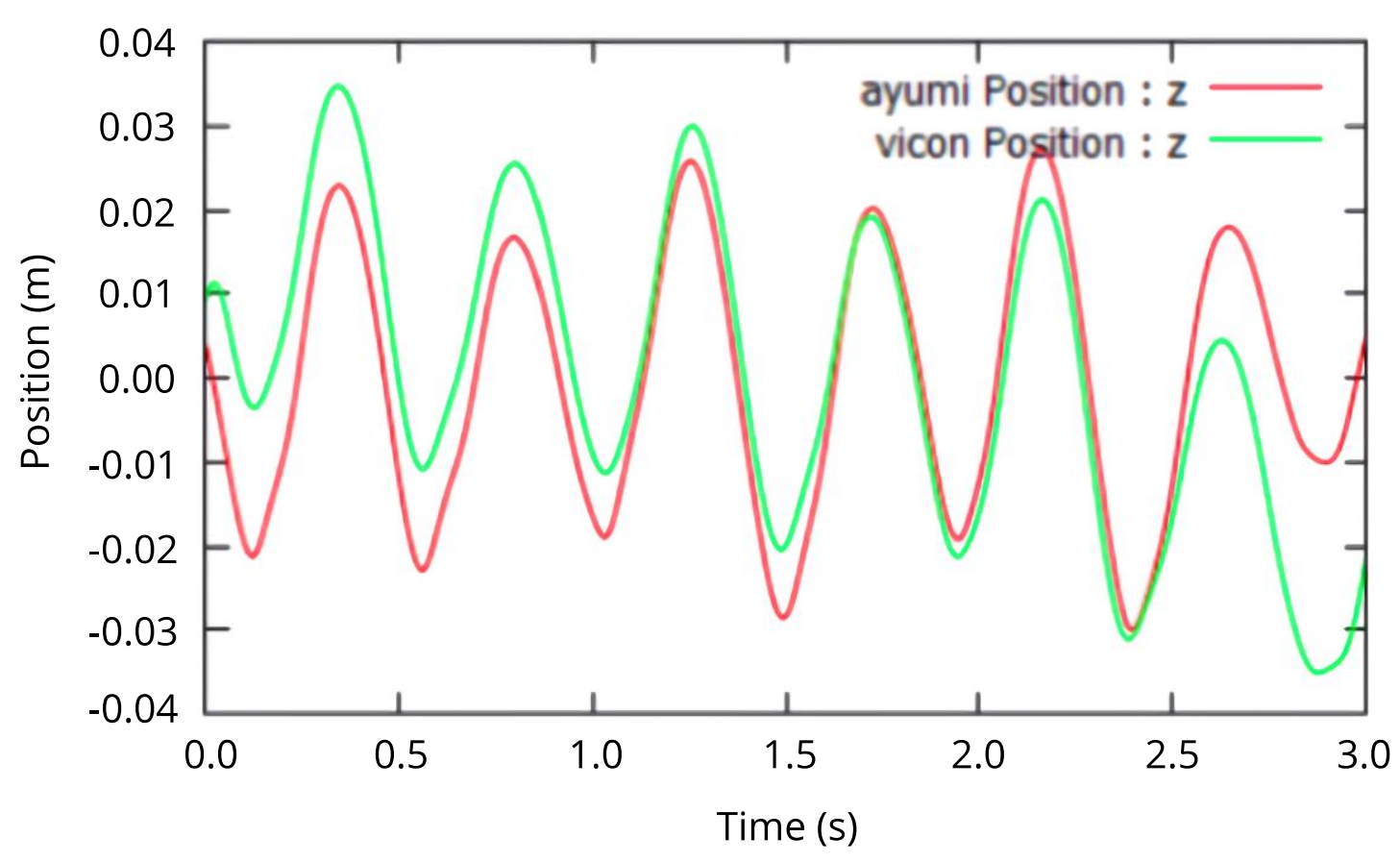

Fig. 4. Typical examples of vertical displacement waveforms from AYUMI EYE and VICON

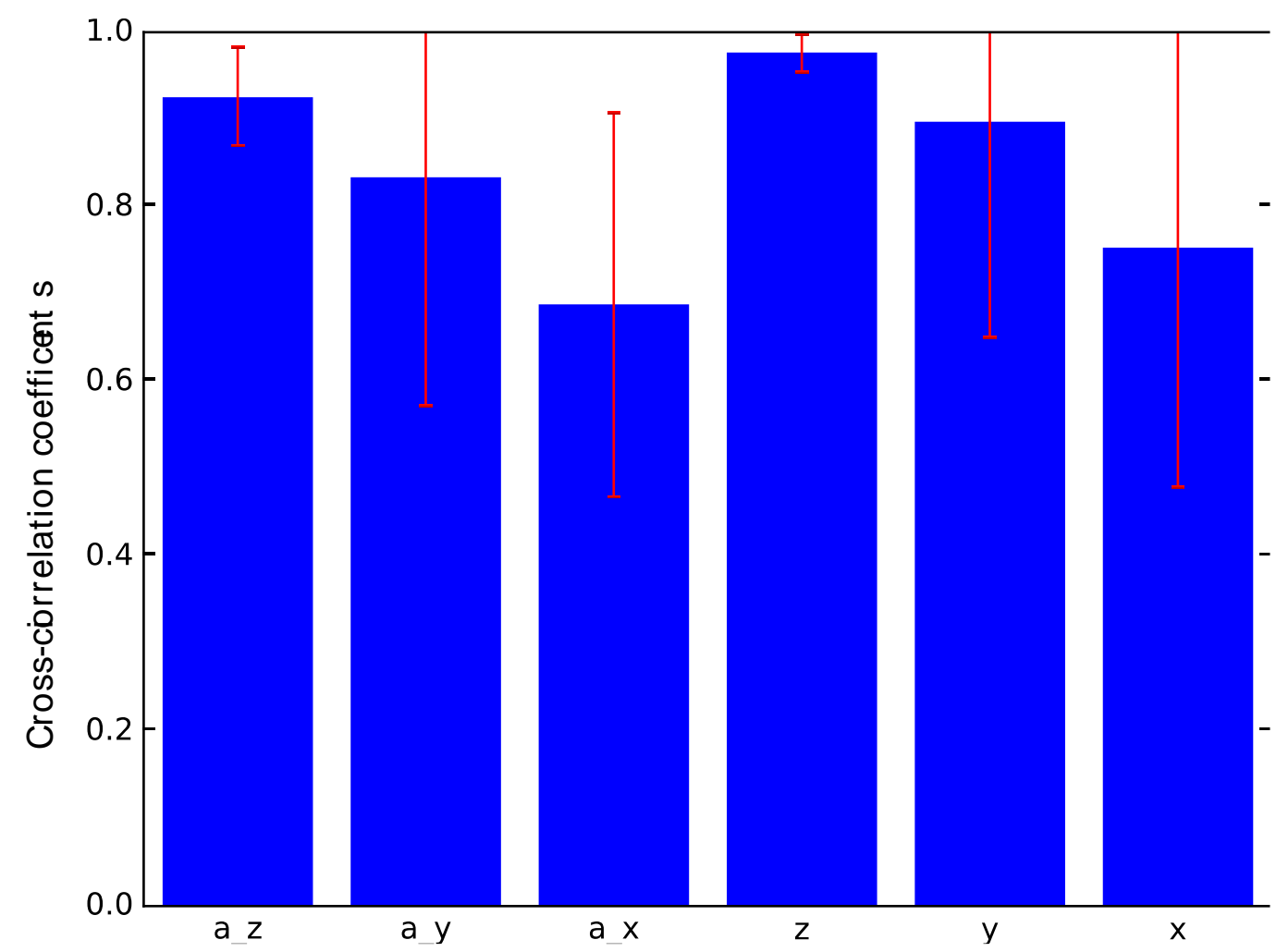

Fig. 5. Mean and SD values of the cross-correlation coefficients computed by comparing AYUMI EYE and VICON for 3D acceleration (vertical axis, a $z=0.926 \pm 0.055$; lateral axis, a $\_y=0.834 \pm 0.259$;

longitudinal axis, a_x $=0.689 \pm 0.219$ ) and $3 \mathrm{D}$ displacement (vertical axis, $\mathrm{z}=0.977 \pm 0.021$; lateral axis, $\mathrm{y}$ $=0.898 \pm 0.245$; longitudinal axis, $\mathrm{x}=0.754 \pm 0.272$ ) 


\subsection{Durations of subsequent stride cycles of AYUMI EYE and force plate}

The number of gait cycle data points obtained by the force plate depended on the gait conditions, and the anterior-tilt walking with the largest stride length had the smallest number of data points [normal walking, 12; anterior-tilt walking, 10; hemiplegic walking, 38; shuffling walking, 39 (Fig. 6)].

Figure 6 compares the durations of the subsequent stride cycles for all the gait trials, for which the corresponding $\mathrm{CC}$ was calculated $(\mathrm{r}=0.896, \mathrm{p}<0.05)$. In normal walking, the $\mathrm{CC}$ computed by comparing each duration of the subsequent stride cycle was very high $(r=0.977, p<0.05$; Fig. 7).

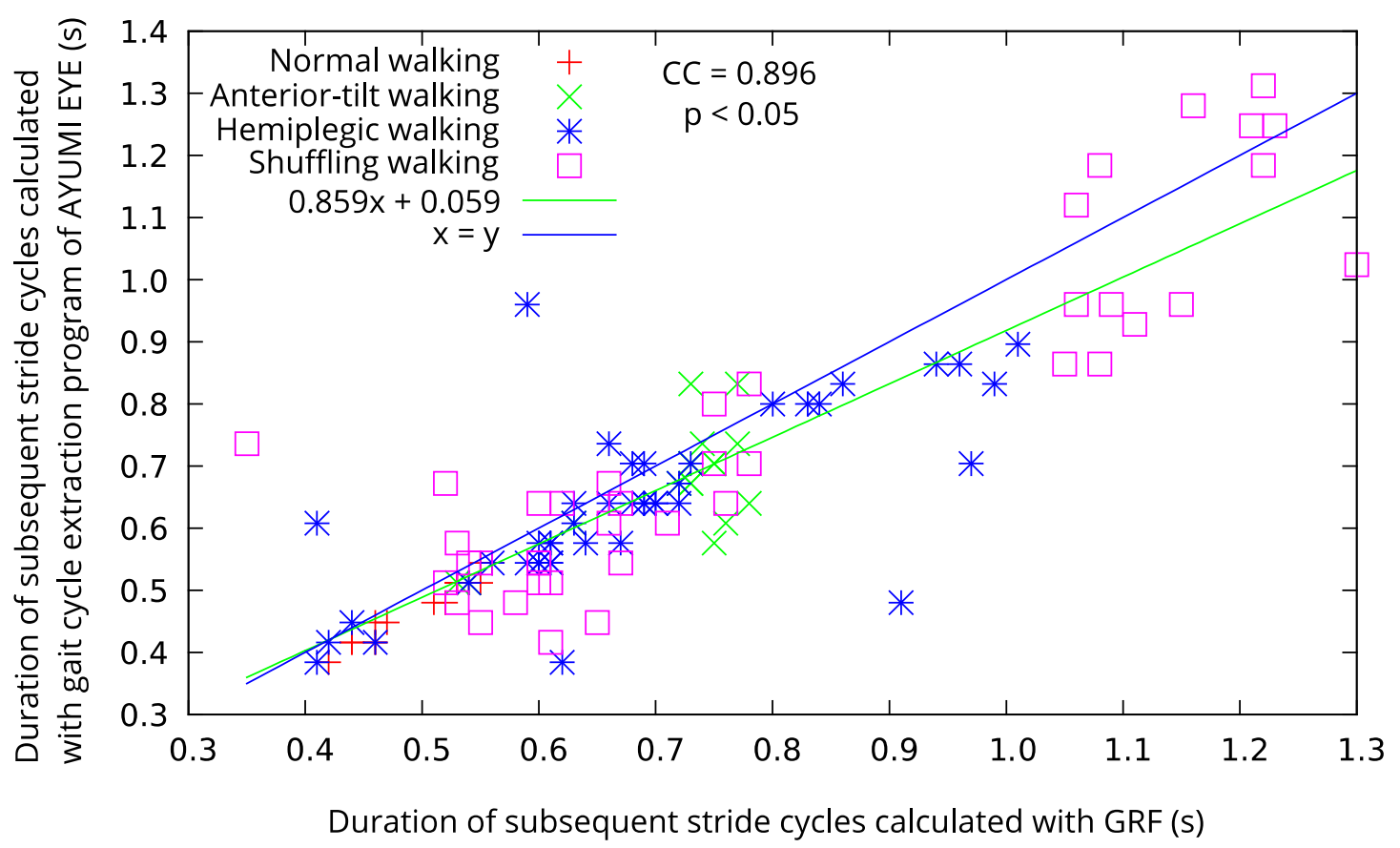

Fig. 6. Comparison of the durations of subsequent stride cycles between the prediction gait cycle extraction program of AYUMI EYE and observation with GRF in all the gait trials. The blue line represents the case where the prediction is equal to the data. 


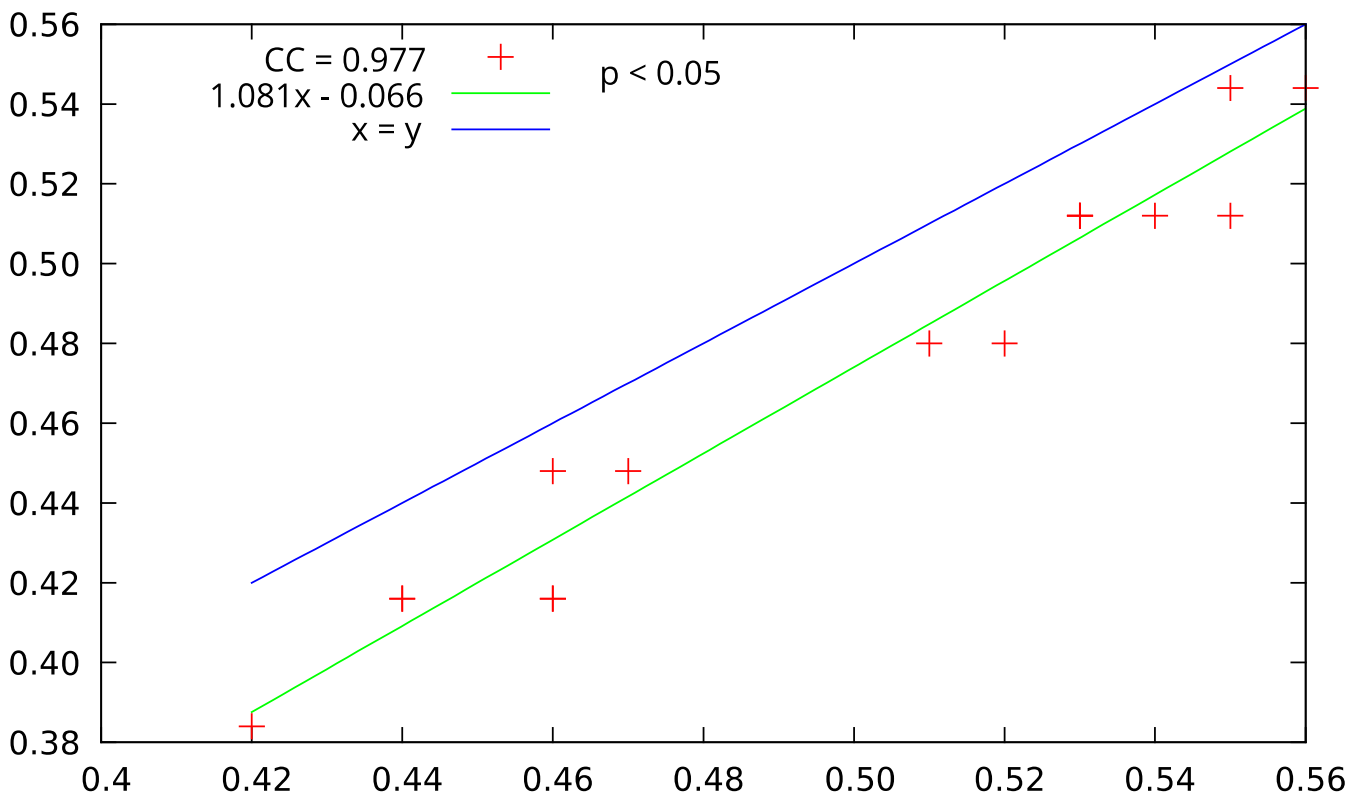

Duration of subsequent stride cycles calculated with GRF (s)

Fig. 7. Comparison of the durations of subsequent stride cycles between the prediction gait cycle extraction program of AYUMI EYE and observation with GRF in normal walking. The blue line represents the case where the prediction is equal to the data.

\section{DISCUSSION}

The AYUMI EYE is a gait analysis system that combines a triaxial accelerometer with an iOS application for easy measurement and data processing, making it easier to introduce it into clinical practice than existing accelerometers [5-13]. Therefore, the reliability and validity of the AYUMI EYE measurement results should be examined in detail. In this study, the researchers investigated the measurement accuracy of the AYUMI EYE hardware and the gait cycle extraction program by simultaneously measuring and comparing the data obtained from the AYUMI EYE, the GRF, and the marker displacements of the VICON.

In this study, the accuracy of the AYUMI EYE measurements and gait cycle extraction program was determined to be reasonable if the acquired data using the module provided sufficient $\mathrm{CC}$ with the data of the VICON and force plate. The CC value is a measure of the similarity between two signal shapes or waveforms; the $\mathrm{CC}$ normalizes the correlation to a dimensionless value in the range of -1 to +1 . A value closer to one for two waveforms indicates a higher degree of coincidence [18]. The acceleration data obtained by the AYUMI EYE showed waveforms similar to those obtained by the VICON for various walking patterns. In particular, very high CCs were obtained in the vertical and lateral directions, both for acceleration and positional data. Similarly, the durations of the subsequent stride cycles calculated using the AYUMI EYE were in good agreement with the results from the force plate. Thus, the measurement 
accuracies of the AYUMI EYE hardware and gait cycle extraction program were determined to be useful for gait analysis and sufficient for evaluations in various walking situations.

By contrast, the $\mathrm{CC}$ of the acceleration data $(0.689 \pm 0.219)$ and position data $(0.754 \pm 0.272)$ in the longitudinal axis were slightly lower than those in the vertical and lateral axes. To compare the waveforms of acceleration and displacement during walking, the fact that the first-order integration of the acceleration waveform is the velocity and the second-order integration is the displacement was utilized. To obtain the velocity and displacement from the digital acceleration data, the data at each gait cycle should be repeatedly corrected and integrated, which is considered to be poorly reproducible [22]. In this study, even the vertical position data, which had good agreement with the waveforms, had some misalignments, as observed after $2.5 \mathrm{~s}$ in Fig. 4. Hence, these effects may have been affected more strongly along the longitudinal axis than the vertical and lateral axes. In addition, the sampling frequency of $31.25 \mathrm{~Hz}$ for the accelerometer built into the AYUMI EYE and $100 \mathrm{~Hz}$ for the VICON and force plate may have influenced the errors in the acquired data. A comparison of the durations of subsequent stride cycles demonstrates that the difference in sampling frequency between the devices may be because the CCs obtained for all walking patterns were lower than those obtained for normal walking only. In previous studies comparing accelerometers with existing gait analysis equipment, either the sampling frequency was aligned or only normal gait was compared [14] [16]. In this study, it was suggested that atypical features that do not occur in normal walking could not be acquired by an accelerometer with a small sampling frequency in anterior-tilt, hemiplegic, and shuffling walking patterns. In some cases, the filtering performed to remove noise from the acceleration data may have affected the results [23] [24].

The first limitation of this study is the validity of comparing the accuracies of the AYUMI EYE measurements and gait cycle extraction program using only the CC (waveform agreement). Several papers have revealed the existence of systematic bias by comparing the accelerometer with another analytical instrument [23] [25], and this should be clarified in the present study. In addition, this study did not define the timing of ground contact and take-off in the acceleration waveform but only compared the durations of the subsequent stride cycles estimated by the AYUMI EYE program, which extracts the gait cycle using the periodicity of the acceleration waveform, with those of the force plate. Thus, it is necessary to analyze further these limitations, evaluate in detail the extent to which the acceleration and position data coincide and the amount of error that exists in the acceleration waveform, and study in detail the parts of the acceleration waveform corresponding to the timings of the ground contact and take-off defined by the force plate. Furthermore, although the instrumental reproducibility of the AYUMI EYE has been shown to be good for this measurement [17], noise and error may have had an effect on the smoothing process of the data of each instrument and the synchronization between instruments.

Despite these limitations, it is significant that the researchers were able to propose the AYUMI EYE as a new gait evaluation tool. In the future, the researchers will clarify the usefulness of the AYUMI EYE as a tool through cross-sectional and longitudinal research. 


\section{CONCLUSIONS}

The AYUMI EYE and VICON showed good agreement for both the acceleration and displacement data. The durations of subsequent stride cycles calculated using the AYUMI EYE and GRF were in good agreement, and their accuracies for these results were sufficient for evaluations in various walking situations. Further, although this study assumed multiple gait conditions, it is still difficult to consider completely the gaits of elderly participants, children, and people with diseases because some of the gait patterns in this study, such as anterior-tilt walking, were simulated by healthy adults. It has been suggested that sufficient investigation is necessary to evaluate abnormal gait by accelerometers [16]. This will be a subject in future research.

\section{ACKNOWLEDGMENTS}

The authors would like to thank Professor Sumiko Yamamoto of the International University of Health and Welfare, Department of Assistive Technological Science Graduate School, for technical assistance.

\section{REFERENCES}

[1] Q. Sun, M. K. Townsend, O. I. Okereke, O. H. Franco and F. Grodstein, "Physical Activity at MidLife in Relation to Successful Survival in Women at Age 70 Years and Older," Arch Intern Med, vol. 170, no. 2, p. 194-201, 2010. doi:10.1001/archinternmed.2009.503.

[2] S. Studenski, S. Perera, K. Patel, C. Rosano, K. Faulkner, M. Inzitari, J. Brach, J. Chandler, P. Cawthon, E. B. Connor, M. Nevitt, M. Visser, S. Kritchevsky, S. Badinelli, T. Harris, A.B. Newman, J. Cauley, L. Ferrucci and J. Guralnik, "Gait Speed and Survival in Older Adults," JAMA, vol. 305, no. 1, pp. 50-58, 2011. doi:10.1001/jama.2010.1923.

[3] H. Miyahara and J. Takeshita, "The relation of exercise performance with healthy life span in the community dwelling elderly: a three-year follow-up study," J Jpn Phys Ther Assoc, vol. 31, p. 155159, 2004. doi:10.15063/rigaku.KJ00001021065 (in Japanese).

[4] "AYUMIEYE," Waseda Elderly Health Association Co., Ltd., [Online]. Available: https://ayumieye.com/. [Accessed 30 April 2020].

[5] J. J. Kavanagh and H. B. Menz, "Accelerometry: a technique for quantifying movement patterns during walking," Gait \& Posture, vol. 28, no. 1, pp. 1-15, 2008. doi:10.1016/j.gaitpost.2007.10.010.

[6] H. Osaka, K. Shinkoda, S. Watanabe, D. Fujita, K. Kobara, Y. Yoshimura, T. Ito and T. Suehiro, "Association between trunk acceleration during walking and clinically assessed balance in patients with stroke," NeuroRehabilitation, vol. 41, no. 4, pp. 783-790, 2017. doi:10.3233/NRE-172171. 
[7] H. Osaka, K. Shinkoda, S. Watanabe, D. Fujita, H. Ishida, K. Kobara, Y. Yoshimura and T. Ito, "Validity of Evaluation Index Utilizing Three Components of Trunk Acceleration during Walking," J. Phys. Ther. Sci., vol. 25, no. 1, pp. 81-84, 2013. doi:10.1589/jpts.25.81.

[8] R. Moe-Nilssen, "Test-retest reliability of trunk accelerometry during standing and walking," Arch Phys Med Rehabil, vol. 79, no. 11, pp. 1377-1385, 1998. doi:10.1016/S0003-9993(98)90231-3.

[9] B. Auvinet, G. Berrut, C. Touzard, L. Moutel, N. Collet, D. Chaleil and E. Barrey, "Reference data for normal subjects obtained with an accelerometric device," Gate \& Posture, vol. 16, no. 2, pp. 124134, 2002. doi:10.1016/s0966-6362(01)00203-x.

[10]M. Yamada, "The Assessment of an Abnormal Gait by Gait Parameters derived from Trunk Acceleration in Patients with Osteoarthritis of the Hip - Comparison with Healthy Controls and Criterion-Related Validity," J Jpn Phys Ther Assoc, vol. 33, no. 1, 2006. doi:10.15063/rigaku.KJ000042249 (in Japanese).

[11] M. Yamada, "Relationship between gait disorder and impairments in patients with hip osteoarthritis : Examination by multiple liner regression analysis," J Phys Med, vol. 16, no. 4, 2005 (in Japanese).

[12] T. Asai, "The assessment of an abnormal gait using tri-axial accelerometer in patients with osteoarthritis of the knee," Kobegakuin Journal of Rehabilitation Research, vol. 4, no. 2, 2009 (in Japanese).

[13]H. Osaka, S. Watanabe, D. Fujita, H. Ishida, K. Kobara, Y. Yoshimura, T. Ito and K. Shinkoda, "Appropriate Location of an Accelerometer for Gait Analysis: A Comparative Study Based on Crosscorrelation Coefficients," J Phys Ther Sci, vol. 26, no. 6, pp. 785-789, 2011. doi:10.1589/rika.26.785 (in Japanese).

[14] N. Tanaka, S. Sonoda, T. Muraoka, Y. Tomita and N. Chino, "Reproducibility and validity of gait analysis using portable accelerometer," Jpn. j. rehabil. med., vol. 33, no. 8, pp. 549-553, 1996. doi:10.2490/jjrm1963.33.549 (in Japanese).

[15] M. Henriksen, H. Lund, R. Moe-Nilssen, H. Bliddal and B. Danneskiod-Samsøe, "Test-retest reliability of trunk accelerometric gait analysis," Gait \& Posture, vol. 19, no. 3, pp. 288-297, 2004. doi: 10.1016/S0966-6362(03)00069-9.

[16] K. Takata and M. Abo, "Gait evaluation with a small three-dimensional accelerometer," Tokyo Jikeikai Medical Journal, vol. 119, no. 1, pp. 331-338, 2004 (in Japanese).

[17] T. Ito, "Reproducibility of AYUMI EYE as an evaluation system for walking ability," Journal of the Japan Society for Health and Medicine, vol. 7, no. 2, 2019 (in Japanese).

[18]D. Saha, S. Gard and S. Fatone, "The effect of trunk flexion on able-bodied gait," Gait \& Posture, vol. 27, no. 4, pp. 653-660, 2008. doi:10.1016/j.gaitpost.2007.08.009. 
[19] S. Yamamoto, "Gait Analysis of Patients with Cerebrovascular Disease," J. Phys. Ther. Sci., vol. 17, no. 1, 2002. doi:10.1589/rika.17.3 (in Japanese).

[20] H. Nagasaki, "Geriatric Gait," Journal of the Society of Biomechanisms Japan, vol. 19, no. 3, 1995. doi:10.3951/sobim.19.148 (in Japanese).

[21] D. Winter, Biomechanics and motor control of human movement, 4th ed., New Jersey: John Wiley \& Sons, Hoboken, 2009.

[22] H. Nomiyama, K. Ogata and M. Yasunaga, "Reappearance of Accelerography and Effects of Wedged Insole on the Knee with an Accelerometric Technique," Journal of Orthopaedic and Disaster Surgery, vol. 42, no. 3, pp. 1041-1047, 1993. doi:10.5035/nishiseisai.42.1041 (in Japanese).

[23]D. Steins, I. Sheret, H. Dawes, P. Esser and J. Collett, "A smart device inertial-sensing method for gait analysis," J Biomech., vol. 47, no. 15, pp. 3780-3785, 2014. doi:10.1016/j.jbiomech.2014.06.014.

[24] P. Esser, H. Dawes, J. Collett and K. Howells, "IMU: inertial sensing of vertical CoM movement," J Biomech., vol. 42, no. 10, pp. 1578-1581, 2009. doi:10.1016/j.jbiomech.2009.03.049.

[25] Y. Horimizu, M. Kimoto, Y. Terui, A. Takahashi, T. Fukui and T. Shioya, "Validity of the Center of Mass Assessed by an Accelerometer: Comparison with a 3D Motion Capture System," Journal of Physical Therapy Science, vol. 31, no. 4, pp. 591-596, 2016. doi:10.1589/rika.31.591 (in Japanese). 